\title{
iTimeline: Uma Ferramenta Visual para Identificação e Acompanhamento das Interações Sociais em Ambientes Virtuais de Aprendizagem
}

\author{
Francisco S. Sousa Neto, José Francisco M. Netto, Dhanielly P. R. de Lima \\ Programa de Pós-Graduação em Informática (PPGI) - Universidade Federal do \\ Amazonas (UFAM) - Manaus - A M - Brasil \\ \{francisconeto, jnetto, dhanielly\}@icomp.ufam.edu.br
}

\begin{abstract}
This paper presents a tool called iTimeline (time line intelligent) mediated by Intelligent Agents (AIs), whose main objective is to help teachers who teach classes in a Virtual Learning Environment. The tool to offers teachers information about the interactions of students in form of time line, enabling the monitoring of interactions of students and displaying the information interactional through the AIs that structure the interactions of sudents and graphically displays the teachers. In this way, the teacher can monitor graphics card and chronologically the social interactions, and adopt strategies that promote the teaching and learning.
\end{abstract}

Resumo. Este artigo apresenta uma ferramenta denominada iTimeline (linha do tempo inteligente) mediada por Agentes Inteligentes (AIs), cujo objetivo principal é auxiliar os professores que ministram aulas em um Ambiente Virtual de Aprendizagem. A ferramenta apresentada disponibiliza aos professores as informações acerca das interações dos alunos em forma de linha do tempo, possibilitando o acompanhamento das interações dos alunos e exibindo as informações interacionais por intermédio dos AIs que estruturam as interações dos cursistas e as exibe graficamente aos professores. Desta forma, o professor pode acompanhar gráfica e cronologicamente as interações sociais, e adotar estratégias que promovam o ensino e aprendizagem.

\section{Introdução}

Os Ambientes Virtuais de Aprendizagem (AVA), são plataformas que apoiam o Ensino a Distância (EaD). Esses ambientes têm mostrado sua importância nesta era moderna e tecnológica e tem exigido dos estudiosos uma atenção especial quanto ao enorme número de interações entre os alunos, e como analisar de maneira rápida esse grande volume de dados interacionais.

Algumas ferramentas interativas são utilizadas para facilitar no processo de ensino-aprendizagem e estimular a colaboração e interação entre os participantes de um curso baseado na web [Fuks et al. 2004] e se fazem necessárias no Ensino a Distância. Além disso, tais tecnologias, estão sendo introduzidas há algum tempo nesta modalidade de ensino e aprendizagem virtual. Todavia, é relevante ressaltar que entre essas Tecnologias da Informação e Comunicação (TICs), estão presentes os Ambientes Virtuais de Aprendizagem, que possuem uma carência considerável de ferramentas para subsidiar o mediador na visualização das interações entre os alunos [Neto et. al. 2016]. 
Percebe-se, no entanto, que em diversos momentos os ambientes computacionais propostos para fins educacionais apenas transpõem para o meio virtual um modelo tecnicista de ensino-aprendizagem, muito similar ao que os alunos vivenciam nas salas de aulas tradicionais. Esses ambientes ajudam os alunos a desenvolverem diversas características e habilidades, pois destacam a ênfase educativa na disseminação do ensino-aprendizagem.

Nesse contexto, acompanhar as interações dos alunos nos ambientes virtuais tem se tornado uma tarefa árdua e exaustiva para o professor. Diante desse fator preocupante foi desenvolvido uma ferramenta visual e interativa para auxiliar o professor no monitoramento dos índices interacionais dos participantes no curso, com posse desta ferramenta o professor pode intervir pedagogicamente ou não, assim evitando evasões, e consequentemente promovendo o aprendizado por meio da interação social entre os estudantes.

Diante disso, a proposta deste estudo é o desenvolvimento de um sistema que monitore as interações dos alunos no AVA. As interações poderão ser visualizadas pelo professor a partir de seus dispositivos móveis ou computadores. Dessa maneira, os alunos estarão cada vez mais conectados com os outros, proporcionando a interação o ensino e a aprendizagem entre eles.

A fim de relatar esta pesquisa, este artigo foi dividido em 7 Seções, contando a partir da introdução. A Seção 2 destaca a fundamentação teórica embasando a elaboração da pesquisa. Na Seção 3 as pesquisas e os trabalhos correlatos são mencionados. A Seção 4 apresenta a ferramenta e seu modelo arquitetural. A Seção 5 destaca a metodologia utilizada. A Seção 6 mostra os resultados alcançados. Finalmente, a Seção 7, apresenta as considerações finais.

\section{Fundamentação Teórica}

Este artigo está fundamentado nos conceitos de Data Visualization e métodos de Visualização das Interações em Ambientes Virtuais de Aprendizagem, detalhados a seguir.

\subsection{Data Visualization em AVAs}

Data Visualization é uma das áreas da tecnologia da computação que vem se destacando nos últimos anos por contribuir no monitoramento de grandes bases de dados, e surge como uma metodologia cada dia mais moderna em relação a visualizar e analisar dados. Este método visual envolve a criação de um estudo representativo dos dados.

Segundo Masud et al. (2010), considera visualizações no domínio mais amplo de comunicação e define um modelo capaz de levar em conta o contexto em que visualizações atuam como ferramentas de comunicação, neste sentido, o autor acima mencionado identifica os dados, as informações e um contínuo conhecimento como o elemento chave para uma estrutura que considera a visualização um processo e não um produto.

De acordo com Tervakari et al. (2014), ferramentas analíticas visuais podem ajudar os professores a tomar decisões sobre estratégias pedagógicas possíveis, 
VI Congresso Brasileiro de Informática na Educação (CBIE 2017)

Anais do XXVIII Simpósio Brasileiro de Informática na Educação (SBIE 2017)

orientação educacional, ações e intervenções que podem ser usados para promover e apoiar a participação e a atividade dos alunos.

Entretanto, o objetivo principal de visualizar dados neste trabalho, é o de comunicar e informar de maneira clara e eficiente os professores por meio dos recursos visuais. A metodologia de visualização de dados ajuda os mediadores analisar e raciocinar os dados, verificando a veracidade dos dados complexos de forma mais acessível, compreensível e utilizável.

\subsection{Métodos de Visualização das Interações em AVAs}

$\mathrm{Na}$ literatura é possível identificar alguns trabalhos que analisam as interações entre os alunos nos AVAs, com o propósito de auxiliar o professor no monitoramento e na coordenação de maneira eficiente das interações dos cursistas, a seguir são descritos alguns desses estudos.

Serrão et al. (2011) apresenta um software social que possibilita a criação e articulação de redes sociais online, para os alunos do ambiente Moodle de forma automática por meio dos dispositivos móveis, potencializando as interações entre alunos e professores.

Lima et al (2017) analisa em seu trabalho as interações dos participantes de um Ambiente Virtual de Aprendizagem. No trabalho, são analisadas as ferramentas fórum e mensagem, e após as análises são geradas automaticamente, por intermédio de um Sistema Multiagente, diversas tabelas e sociogramas das interações dos alunos. Em cima desses resultados, são aplicadas e calculadas alguma métricas de Análise de Rede Social (ARS) - densidade, inclusividade e centralidade, que auxiliam por exemplo, na identificação dos alunos mais participativos e menos participativos. Esse trabalho teve como finalidade, auxiliar o professor a compreender as disposições interacionais estabelecidas por seus alunos na disciplina.

Sacerdote e Fernandes (2013) analisam as interações entre os participantes de um Ambiente Virtual de Aprendizagem. Os dados analisados nesta pesquisa, foram coletados das atividades executadas pelos usuários, troca de mensagens e nos fóruns de discussões por meio do método exploratório de Análise de Redes Sociais e utilizou também, os arquivos de registros de atividades individuais geradas pelo ambiente.

Por fim, o trabalho de Romani (2000) que apresenta uma ferramenta denominada InterMap, que utiliza técnicas de visualização de informação para mapear graficamente dados armazenados pelas ferramentas de comunicação: correio, grupo de discussão e bate-papo, do TelEduc, um ambiente para suporte ao ensino/aprendizagem a distância na Web.

\section{Trabalhos Relacionados}

Atualmente na literatura há inúmeros trabalhos que analisam as interações dos estudantes no AVA, ou até mesmo utilizam Sistemas Multiagente em Ambientes Virtuais de Aprendizagem. A seguir são descritos alguns desses trabalhos que embasam nossa proposta.

No trabalho de Gerosa et al. (2003) é descrito uma análise sobre os fóruns educacionais do ambiente AulaNet, onde são analisados aspectos relacionados a 
estruturação do discurso e à categorização das mensagens, servindo como subsídio de informações ao professor. Além disso, no trabalho é apresentado dados qualitativos sobre as discussões no fórum.

O trabalho de Lima (2015), apresenta uma estratégia baseada numa arquitetura multiagente, tendo como objetivo analisar as interações entre os estudantes que ocorrem nos fóruns e nas mensagens de um AVA, os resultados dessas análises são estruturados e apresentados ao professor sob a forma de sociogramas e de tabelas, além disso, o professor recebe alertas via e-mail do sistema, informando quais os alunos com baixa ou nenhuma interação.

Já Bakharia e Dawson (2011), apresentam uma ferramenta denominada SNAPP que tem como objetivo analisar a interação no fórum entre os estudantes de um curso on-line e assim proporcionar uma representação visual de fácil interpretação da organização do grupo social nos fóruns. A ferramenta possibilita visualizar a evolução da interação entre os participantes ao longo do tempo e anotar os principais eventos que ocorrem ao longo desta linha do tempo. Esse recurso é útil em termos de monitoramento da evolução da rede.

Jaques (1997), descreve um experimento utilizando uma arquitetura multiagente para realizar o monitoramento das ferramentas de comunicação em um ambiente de Ensino a Distância. Este sistema analisa as discussões que se encontram em andamento nas ferramentas de lista de discussão, chat e newsgroup, disponibilizando ao professor informações estatísticas (percentual de participação dos alunos e número de mensagens trocadas), e identificando possíveis associações nas interações, tais como, tópicos e subtópicos que interessam ao aluno e aos grupos de alunos que interagem mais entre si.

Soller (2001), proporciona uma melhor compreensão da interação do grupo e orienta a melhor forma de apoiar o processo de aprendizagem colaborativa. Todavia, propõe um quadro de princípios para o estudo e análise da interação de grupo que é construído através da combinação de diferentes aspectos e questões de colaboração e aprendizagem.

Bassani (2006), faz uma reflexão sobre o acompanhamento das interações em Ambientes Virtuais de Aprendizagem, focalizando-se na modelagem e implementação de uma ferramenta computacional capaz de mapear as interações potencializadas nestes ambientes.

Mora et al. (2014), fazem uma representação visual das interações para melhor compreensão da interação de grupo e determinar a melhor forma de apoiar o processo de aprendizagem colaborativa. Neste sentido, propõem princípios para o estudo e a análise da interação de grupo, que é construído através da combinação de diferentes aspectos e questões de colaboração, aprendizagem e avaliação.

\section{A Ferramenta}

$\mathrm{Na}$ área de Inteligência Artificial existem várias abordagens para solucionar inúmeros problemas, este trabalho utiliza a abordagem de Sistemas Multiagente (SMA) para auxiliar o professor de um AVA a compreender e visualizar as interações estabelecidas por seus alunos nas ferramentas mensagem e fórum de discussão da disciplina. No qual, 
VI Congresso Brasileiro de Informática na Educação (CBIE 2017)

Anais do XXVIII Simpósio Brasileiro de Informática na Educação (SBIE 2017)

foram desenvolvidos quatro agentes que constituem o SMA deste trabalho. O papel desempenhado por cada agente é descrito no Quadro 1.

Quadro 1. Agentes e os seus Papéis no Sistema.

\begin{tabular}{|l|l|}
\hline \multicolumn{1}{|c|}{ Agente } & \multicolumn{1}{c|}{ Papéis } \\
\hline Agente Coletor & $\begin{array}{l}\text { Realiza consulta no banco de dados, verificando as interações sociais entre os } \\
\text { alunos ocorridas no AVA; }\end{array}$ \\
\hline Agente Construtor & $\begin{array}{l}\text { Atualiza os dados das interações dos alunos no ambiente de aprendizagem e } \\
\text { realiza a construção dos gráficos na linha do tempo. }\end{array}$ \\
\hline Agente Timeline & $\begin{array}{l}\text { Constrói uma linha do tempo e apresenta as informações do dia em que } \\
\text { ocorreram, como por exemplo: data e quantidade das interações dos alunos } \\
\text { que serão visualizadas na linha do tempo. }\end{array}$ \\
\hline Agente Email & $\begin{array}{l}\text { Notifica o professor via e-mail sobre as interações dos alunos que estão } \\
\text { ocorrendo no AVA, disponibilizando as informações necessárias e, } \\
\text { permitindo ao professor realizar as intervenções . }\end{array}$ \\
\hline
\end{tabular}

A ferramenta iTimeline está sob a forma de um plug-in, integrado ao Moodle. Em que o professor ao acessar o curso, tem a sua disposição à ferramenta dinâmica e interativa. Porém, para que ela seja visível no ambiente, é necessário ativá-la por intermédio da funcionalidade, ativar edição disponível dentro do próprio Moodle. A ferramenta apresenta ao professor, uma representação visual em forma de grafos e gráficos, que são projetadas com o auxílio dos agentes que coletam e estruturam os dados em forma de linha do tempo. Para um melhor entendimento é apresentada a Figura 1.

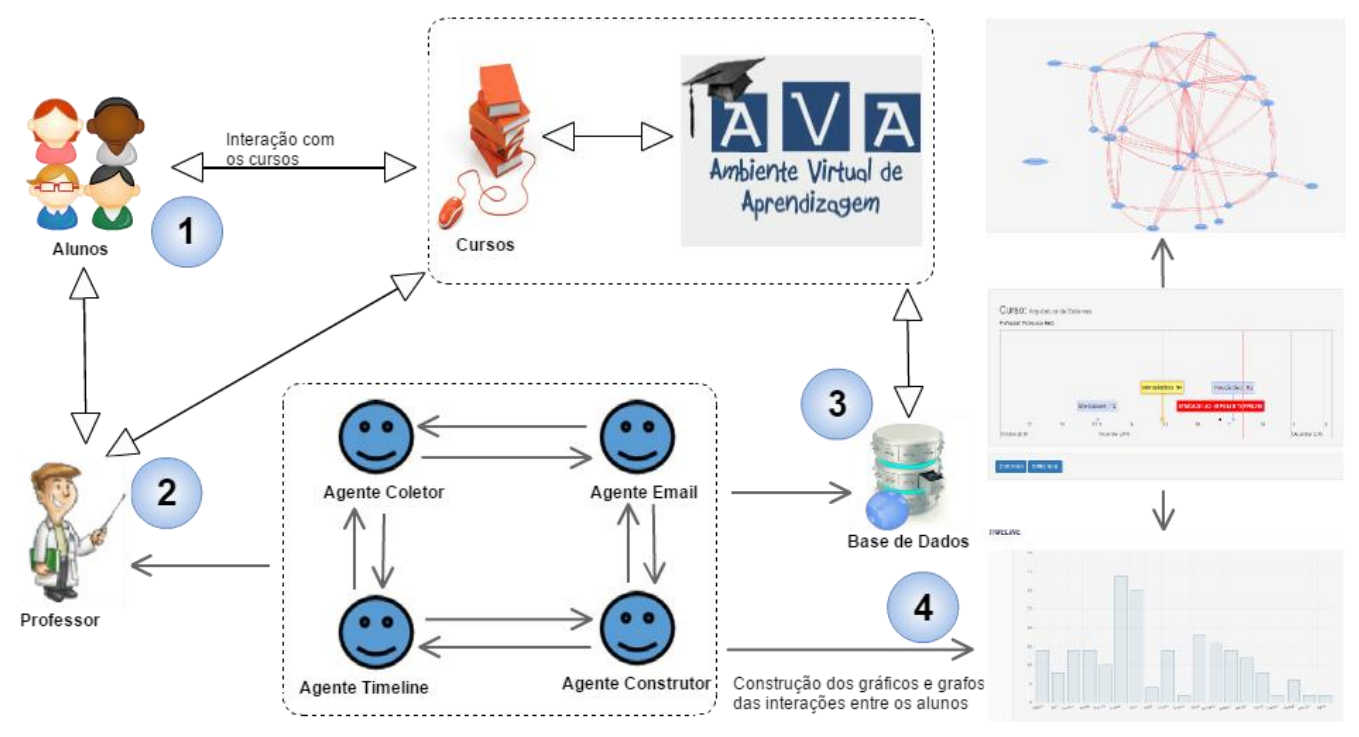

Figura 1: Visão arquitetural do sistema.

De acordo com a Figura 1, pode-se observar que o sistema iTimeline possui 4 agentes inteligentes, que são responsáveis por identificar e coletar no banco de dados as informações referentes as interações dos alunos, e após obtidos esses dados, os agentes caracterizam as interações dos alunos, e as disponibiliza ao professor por meio dos recursos gráficos que o sistema disponibiliza do curso, além desses recursos o sistema envia ao professor por e-mail a situação dos alunos indicando o quadro interativo dos mesmos no ambiente de aprendizagem. 
Os agentes inteligentes da iTimeline foram desenvolvidos em JAVA e modelados segundo a metodologia MASE. Essa metodologia objetiva o ciclo de vida do desenvolvimento do sistema e permite ao desenvolvedor de um SMA construir o passoa-passo, partindo de um conjunto inicial de requisitos até alcançar as fases de análise, projeto e implementação, segundo o trabalho (DeLoach e Wood, 2001).

\section{Metodologia Utilizada}

Para conceber um conjunto de dados representados (recursos visuais) que auxilie o professor na sua ação mediadora, é preciso compreender como ocorrem as interações dos alunos no ambiente. Técnicas de visualização de dados podem auxiliar na interpretação dos diversos perfis dos cursistas, a partir da identificação dos dados gerados no AVA-Moodle, em suas ferramentas síncronas e assíncronas que proporcionam a interação e a comunicação, possibilitando identificar comportamentos que indicam possíveis evasões ou reprovações.

Dessa maneira, torna-se possível propor um conjunto de fases a serem implementadas, a fim de promover melhorias pedagógicas, auxiliando o professor no acompanhamento das interações de seus alunos e, consequentemente, que essa mediação docente reflita em cursistas mais engajados na sua formação educacional.

Para desenvolver o trabalho proposto, foram estruturadas as seguintes fases:

I. Primeira fase - realização de uma revisão bibliográfica para identificar os trabalhos relacionados com a pesquisa, caracterizando assim, o estado da arte das pesquisas referente aos trabalhos relacionados às interações sociais entre os estudantes, a utilização de técnicas de Data Visualization e de Agentes Inteligentes em AVA.

II. Segunda fase - realização do desenvolvimento e implementação da ferramenta, utilizando como base os dados obtidos na fase anterior. Também foi realizada uma pesquisa de campo, onde foram coletados os dados interacionais de uma turma real em um AVA, esses dados foram organizados e analisados pelos autores e pela ferramenta.

III. Terceira fase - realização de testes com a ferramenta implementada. Os dados organizados pela ferramenta passaram por análises para a geração de um modelo baseado em uma linha do tempo, esse modelo representa visualmente as interações entre os alunos.

Foram realizados testes em uma turma simulada e também em uma turma real. Os testes simulados possibilitaram projetar a arquitetura, que por sua vez passou por algumas melhorias e foi adaptada para situações reais que apareceram no desenvolvimento desta pesquisa. Além disso, foi realizado um Estudo de Caso com a ferramenta em um curso de matemática que possuía 35 alunos. Essas informações serão discutidas em detalhes na próxima seção.

\section{Resultados e Discussões}

Foram realizados diversos experimentos com a ferramenta, a fim de torná-la mais usual para o contexto real. Sendo assim, realizou um Estudo de Caso em um curso de Matemática do Instituto Federal do Acre (IFAC), o curso contava com cerca de 35 
VI Congresso Brasileiro de Informática na Educação (CBIE 2017)

Anais do XXVIII Simpósio Brasileiro de Informática na Educação (SBIE 2017)

alunos com perfis diferentes e de diversas localidades. O AVA-Moodle esteve disponível no servidor do Laboratório de Sistemas Inteligentes do Instituto de Computação (IComp) da Universidade Federal do Amazonas (UFAM).

O professor podia interagir com a ferramenta, identificando os dias em que ocorreram as interações e assim acompanhando cronologicamente a dinâmica interacional que acontecia em tempo real no ambiente. É percebível também na Figura 2 , que as interações aconteciam antes de finalizar a data limite para a postagem da atividade.

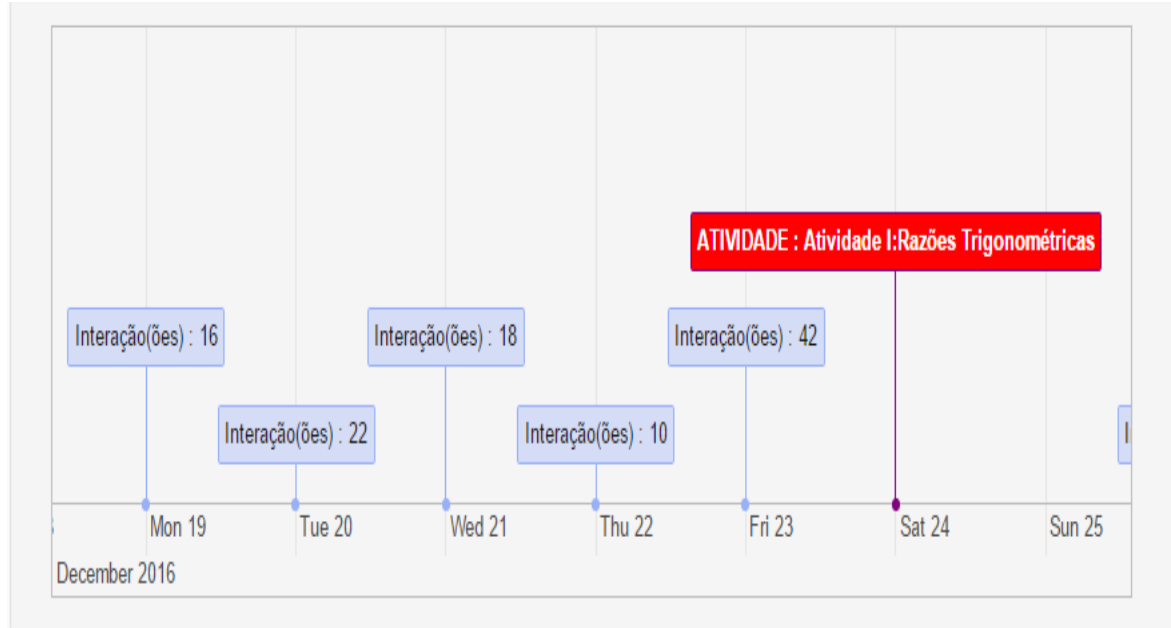

Figura 2: Linha do tempo das interações.

O professor podia analisar as interações dos alunos que ocorriam nos fóruns e nas mensagens do AVA e, solicitar a ferramenta o estado atual das interações no ambiente. A Figura 3 (lado esquerdo) apresenta o grafo das interações da $1^{\text {a }}$ semana, ilustrando ao professor que o nível de interações estava baixo. Todavia, para melhorar o índice de interações entre os alunos, o professor se comunicou com os mesmos que apresentavam baixo nível de interação, notificando-os por meio de e-mail no AVA.

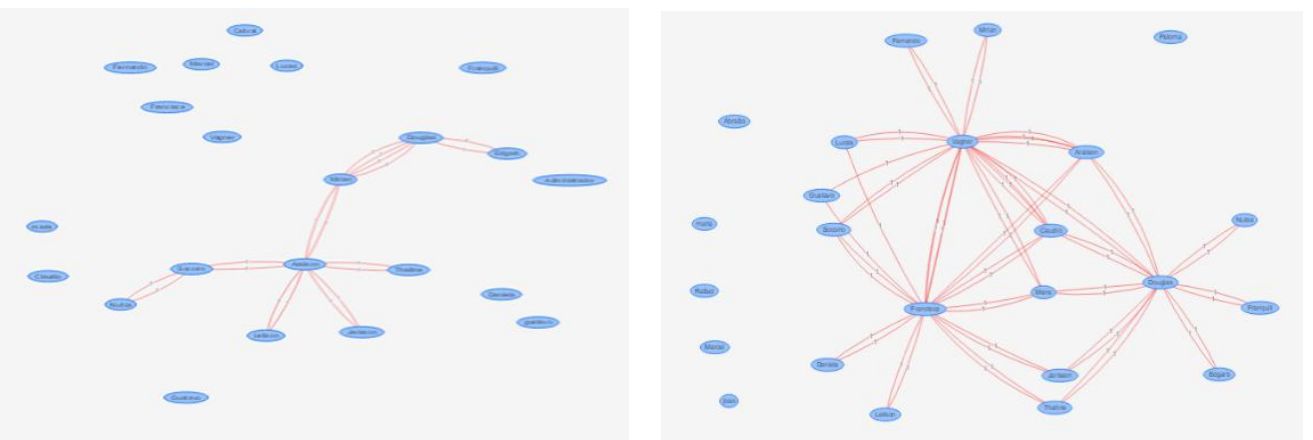

Figura 3: Grafo das interações $1^{\mathrm{a}}$ semana (Lado esquerdo) Grafo das interações 2a semana (Lado Direito).

Os resultados da segunda semana alcançaram uma evolução em comparação aos resultados da primeira semana. Foi possível verificar alguns alunos que já estavam mais participativos no ambiente e interagindo, devido o incentivo do professor.

Também é possível analisar na Figura 3 as interações dos alunos em forma de grafo interacional, que foi gerado no dia escolhido pelo professor. Diante dos resultados identificados, o professor percebeu que as interações da turma cresceram 
consideravelmente em relação aos resultados da primeira semana. A seguir na Figura 4 apresenta quantitativamente as interações dos alunos, possibilitando ao professor analisar as interações de seus alunos individualmente.

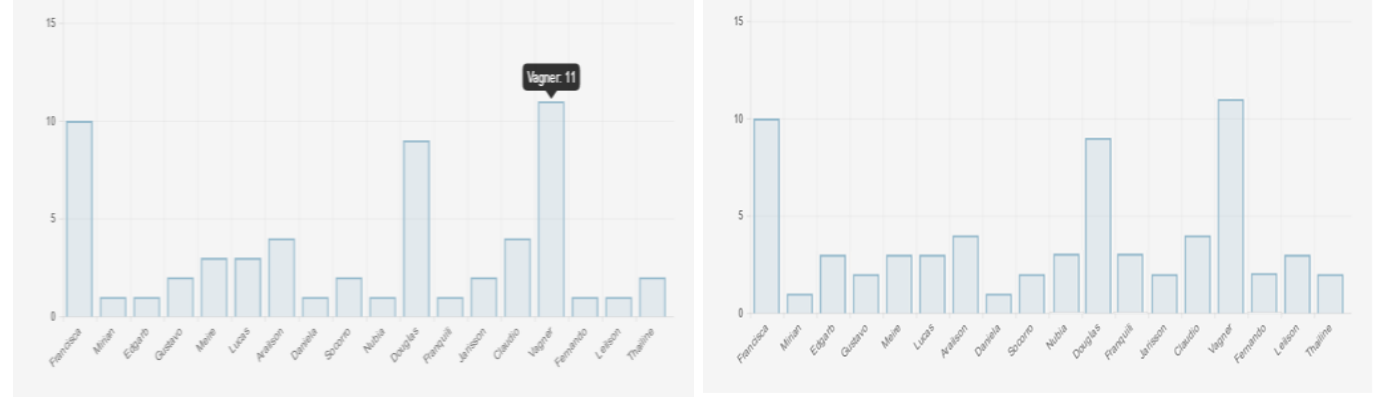

Figura 4: Gráfico das interações 1ª semana (Lado Esquerdo) Gráfico das interações 2a semana (Lado Direito).

Os resultados obtidos foram relevantes, pois a ferramenta auxiliou o professor na identificação dos alunos que apresentavam nenhuma ou pouca interação no ambiente, possibilitando-lhe aplicação de medidas e estratégias pedagógicas e promovendo a integração dos alunos que não estavam participativos. $\mathrm{O}$ uso dos agentes inteligentes e suas características possibilitou um melhor desempenho no monitoramento dos dados, como por exemplo: no envio do e-mail, construção da linha do tempo, simplificando o trabalho do professor no acompanhamento e na análise das interações.

Os resultados obtidos fundamentam a hipótese de que a ferramenta iTimeline auxiliou de forma colaborativa os professores no processo de ensino e na aprendizagem. As atividades de colaboração como a construção de atividade em grupo ou a participação dos alunos nos fóruns e nas mensagens, puderam ser efetivadas pelo professor com o auxílio da ferramenta.

\section{Conclusões}

A abordagem aqui detalhada apresenta sua relevância ao utilizar técnicas de Data Visualization e Sistemas Multiagente e a contribuir com essas áreas do conhecimento no contexto dos Ambientes Virtuais de Aprendizagem. Atualmente, precisa-se de Sistemas Multiagente que impulsionem o amadurecimento de novas metodologias voltadas para visualização de dados nos ambientes virtuais.

A pesquisa desenvolvida aborda a representação visual das interações dos estudantes no AVA, cujo objetivo é auxiliar o professor no acompanhamento do processo de aprendizagem de seus alunos. A ferramenta proporciona a identificação das interações dos alunos no AVA, tornando possível acompanhar e intervir pedagogicamente, além de promover a inter-relação dos alunos e favorecer o processo de ensino-aprendizagem.

Para a efetivação da ferramenta, dois experimentos foram realizados, a ferramenta apresentou-se promissora e eficaz para o acompanhamento das interações dos alunos em Ambientes Virtuais de Aprendizagem. A ferramenta contribuiu positivamente diante dos resultados adquiridos, atendendo as expectativas esperadas 
VI Congresso Brasileiro de Informática na Educação (CBIE 2017)

Anais do XXVIII Simpósio Brasileiro de Informática na Educação (SBIE 2017)

para visualizar dados, por intermédio de técnicas de Data Visualization, atingindo objetivo desta pesquisa.

Como trabalhos futuros, pretende-se expandir a ferramenta, como por exemplo, elaborar uma versão mobile e integrar métricas de redes sociais para estudar as relações sociais estabelecidas na disciplina e em cima disso, elaborar dados de saída mais precisos. Por fim, pretende-se realizar mais testes com a ferramenta e elaborar um estudo de caso mais detalhado e com um tempo de execução maior.

\section{Referências}

Bakharia, Aneesha, \& Shane Dawson. (2011). SNAPP: A Bird's-Eye View of Temporal Participant Interaction. International Conference on Learning Analytics and Knowledge LAK'11, ACM, Banff, Alberta.

Bassani, Patrícia B. S. (2006). Mapeamento das Interações em Ambiente Virtual de Aprendizagem: Uma Possibilidade para Avaliação em Educação a Distância. Tese de Doutorado, UFGS. Porto Alegre-RS.

Deloach, S. A.; Wood, M. (2001). "Developing Multiagent Systems with AgentTool". In: Proceedings of Lecture Notes in Artificial Intelligence. Springer - Verlag. Berlim.

Fuks, H., Gerosa, M. A., Raposo, A. B., \& de Lucena, C. J. P. (2004). O Modelo de Colaboração 3C no Ambiente AulaNet. Informática na Educação: Teoria \& Prática, 7(1).

Gerosa, M.A., Pimentel, M.G., Fuks, H. \& Lucena, C.J.P. (2003), Coordenação de Fóruns Educacionais: Encadeamento e Categorização de Mensagens, XIV Simpósio Brasileiro de Informática na Educação, Rio de Janeiro-RJ.

Jaques, P. A. (1997). Um Experimento com Agentes de Software para Monitorar a Colaboração em Aulas Virtuais. Revista Brasileira de Informática na Educação, Comissão Especial de Informática na Educação, Rio Grande do Sul.

Lima, D. P. R. de; Netto, J. F. M.; Bremgartner, V. (2017) Applying Social Network Analysis in a Course Supported by a LMS: Report of a Case Study. In Frontiers in Education (FIE 2017), Indianápolis - USA.

Lima, Dhanielly Paulina Rodrigues de. (2015). Um Sistema Multiagente de Identificação e Caracterização de Relações Sociais de Alunos em um Ambiente Virtual de Aprendizagem. Dissertação de Mestrado. UFAM, Manaus-AM.

Masud, L., Valsecchi, F., Ciuccarelli, P., Ricci, D., \& Caviglia, G. (2010). From Data to Knowledge-Visualizations as Transformation Processes Within the DataInformation-Knowledge Continuum. In Information Visualisation (IV), 2010 14th International Conference (pp. 445-449). IEEE.

Mora, N., Caballé, S., Daradoumis, T., Gañán, D.; Barolli, L. (2014). Providing Cognitive and Social Networking Assessment to Virtualized Collaborative Learning in Engineering Courses. In: Intelligent Networking and Collaborative Systems (INCoS), International Conference on. IEEE, p. 463-468.

Neto, F.S.S, Netto, J.F.M. Lima, D. P. R. (2016) Análise das Interações Sociais entre os 
VI Congresso Brasileiro de Informática na Educação (CBIE 2017)

Anais do XXVIII Simpósio Brasileiro de Informática na Educação (SBIE 2017)

Participantes de um Curso EaD: Uma Revisão Sistemática da Literatura. XXIV Simpósio Brasileiro de Sistemas Colaborativos (SBSC). Porto Alegre-RS.

Romani, L. A. S. (2000) "InterMap: Ferramenta para Visualização da Interação em Ambientes de Educação a Distância na Web". Dissertação de Mestrado. UNICAMP.

Sacerdote, H. C. S, Fernandes, J. H. C. (2013). Investigating Interactions in a Virtual Learning Environment through Social Network Analysis. InCID: R. Ci. Inf. e Doc., Ribeirão Preto, v. 4, n. 1, p. 129-146.

Serrão T.; Braz, L. M.; Pinto, S. C. e Clunie, G. (2011) “Construção Automática de Redes Sociais Online no Ambiente Moodle”. In: XXII Simpósio Brasileiro de Informática na Educação (SBIE). Aracaju, Brasil.

Soller. A. (2001) Supporting Social Interaction in an Intelligent Collaborative Learning System. International Journal of Artificial Intelligence in Education (IJAIED), Pittsburgh pp.40-62.

Tervakari, A. M., Silius, K., Koro, J., Paukkeri, J., \& Pirttila, O. (2014). Usefulness of Information Visualizations Based on Educational Data. In Global Engineering Education Conference (EDUCON), 2014 IEEE (pp. 142-151). IEEE. 